\title{
Die Ökonomisierung der Medizin ist in den Arztpraxen angekommen
}

\section{Urs Stoffel}

Dr. med., Mitglied des FMH-Zentralvorstandes, Departementsverantwortlicher Ambulante Versorgung und Tarife

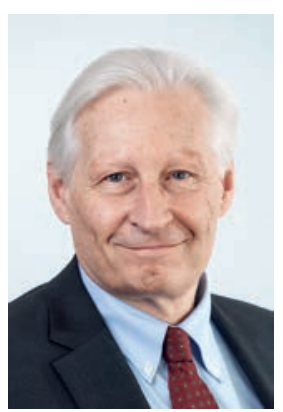

Unter dem Druck des Kostenwachstums im Schweizer Gesundheitswesen taucht in den letzten Jahren immer wieder das Schlagwort «Ökonomisierung der Medizin» auf. Die Schweizerische Akademie der Medizinischen Wissenschaften (SAMW) hat zur Ökonomisierung in der Medizin bereits 2014 ein Positionspapier unter dem Titel «Medizin und Ökonomie - wie weiter?» verfasst. Die SAMW spricht in ihrem Positionspapier unter anderem auch von "Qualitätseinbussen durch fehlgeleitete Effizienzsteigerung» und von «Interessenkonflikten", denen Ärzte und Pflegende im Spannungsfeld zwischen ökonomischen Interessen und dem Patientenwohl ausgesetzt sind.

Die Legitimation für den ökonomischen Druck auf die Medizin wird dabei mit dem Artikel 32 des Krankenversicherungsgesetzes begründet, der die WZW-Kriterien Wirksamkeit, Zweckmässigkeit und Wirtschaftlichkeit als Voraussetzung für die Kostenübernahme im obligatorischen Krankenpflegebereich definiert. Es fällt auf, dass der Begriff der Wirtschaftlichkeit dabei als dritte Forderung der WZW-Kriterien immer mehr in den Fokus gerät. Seit längerem stellt sich die Frage, inwieweit dieser ökonomische Druck, respektive die Kommerzialisierung der Medizin, die Erbringung von medizinischen Leistungen und das ärztliche Handeln der niedergelassenen Ärzteschaft beeinflusst.

Es stellt sich die Frage, inwiefern der ökonomische Druck das ärztliche Handeln der niedergelassenen Ärzteschaft beeinflusst.

Nun liegt erstmals eine repräsentative Studie vor, welche dieser Frage nachgeht und die Auswirkungen der zunehmenden ökonomischen Regulierungen aufzeigt. Sie wurde von Patrick Müller, dem operativen Leiter des Departements Ambulante Versorgung und Tarife, im Rahmen seiner Masterarbeit zum MBA verfasst. Eine Zusammenfassung der Studienergebnisse finden Sie in dieser Ausgabe der SÄZ.

Er konnte in seiner Studie klar nachweisen, dass der ökonomische Druck und die daraus resultierenden Regulierungen tatsächlich zu einer Kommerzialisierung der Medizin führen und auch Auswirkungen auf die Versorgung haben.

Die Zunahme der "Gruppenpraxis» als Versorgungsmodell ist eine Tatsache. Sie wird aber entgegen gängiger Annahmen nicht in erster Linie vom ökonomischen Druck getrieben. Nach wie vor sind es persönliche Gründe, welche bei der Wahl der Praxisform im Vordergrund stehen. Nichtsdestotrotz führen neben fachlichen

\section{Die Befragung legt nahe, dass Massnahmen} zur Stärkung der Attraktivität der Arbeit in der freien Praxis angezeigt sind.

Gründen, vor allem bei den Spezialisten, auch ökonomische Überlegungen dazu, sich für das Modell der Gruppenpraxis zu entscheiden. Dies sicherlich deshalb, weil die besonders teuren Infrastrukturen in einer Gruppenpraxis besser und effizienter genutzt werden können. Die Studie zeigt auch, dass vier von fünf Ärztinnen und Ärzten der Meinung sind, dass das Medizinstudium und die Weiterbildung am Spital oder in der Praxis betriebswirtschaftliche Aspekte beinhalten sollte. Damit ist die Ökonomisierung der Medizin definitiv in den Arztpraxen angekommen.

Auch die Tarifeingriffe des Bundesrates von 2014 und 2018 haben deutliche Spuren hinterlassen und führen zu Anpassungen des Leistungsangebots. Ein grosser Teil der befragten Ärztinnen und Ärzte ist auch der Meinung, dass sich das Arzt-Patienten-Verhältnis verändert hat und viele Patienten heute bereits mit konkreten Forderungen nach Leistungen in die Arztpraxen kommen.

Patrick Müller kommt in seiner Studie zum Schluss, dass die Attraktivität der niedergelassenen ärztlichen Tätigkeit als Folge der zunehmenden politischen Regulierungen und Eingriffe auf Vergütungs- oder Kostenebene abnimmt. Auf Basis seiner Studienergebnisse formuliert er fünf Empfehlungen. Im Vordergrund stehen dabei die Aufnahme von betriebswirtschaftlichen Modulen in der Aus- und Weiterbildung von Ärztinnen und Ärzten und geeignete Massnahmen zur Stärkung der Attraktivität der ärztlichen Tätigkeit in der freien Praxis. 